\title{
Imatinib induces H2AX phosphorylation and apoptosis in chronic myelogenous leukemia cells in vitro via caspase-3/Mst1 pathway
}

\author{
Yan-jun ZHANG ${ }^{1,2}$, Cheng-rong $\mathrm{LU}^{1,}{ }^{*}$, Yan $\mathrm{CAO}^{1,2}$, Yuan $\mathrm{LUO}^{1}$, Rong-feng BAO ${ }^{1}$, Shu YAN ${ }^{1,3}$, Mei XUE ${ }^{4}$, Feng ZHU ${ }^{5}$, Zhe \\ WANG $^{1}$, Lian-ning DUAN ${ }^{1, *}$ \\ ${ }^{1}$ Aviation Medicine Research Lab and ${ }^{4}$ Department of Hematology, General Hospital of Air Force, Beijing 100142, China; ${ }^{2}$ Graduate \\ Student Ministry of Education, Hebei North University, Zhangjiakou 075000, China; ${ }^{3}$ Department of Post-Graduate, China Medical \\ University, Shenyang 110001, China; ${ }^{5}$ Hormel Institute, University of Minnesota, Austin, MN 55912, USA
}

Aim: Histone H2AX is a novel tumor suppressor and its phosphorylation at the C terminus (Ser139 and Tyr142) is required for tumor cell apoptosis. The aim of the present study was to elucidate the mechanisms underlying imatinib-induced C-terminal phosphorylation of $\mathrm{H} 2 \mathrm{AX}$ in chronic myelogenous leukemia cells in vitro.

Methods: BCR-ABL-positive K562 cells were used. Microscopy, Western blotting and flow cytometry were used to study the signaling pathways that regulate imatinib-induced H2AX phosphorylation and the apoptotic mechanisms.

Results: Treatment of K562 cells with imatinib (1-8 $\mu \mathrm{mol} / \mathrm{L})$ induced phosphorylation of H2AX at Ser139 and Tyr142 in time- and dose-dependent manners. In contrast, imatinib at the same concentrations did not affect H2AX acetylation at Lys 5, and the acetylated H2AX maintained a higher level in the cells. Meanwhile, imatinib (1-8 $\mu \mathrm{mol} / \mathrm{L})$ activated caspase- 3 and its downstream mammalian STE20-like kinase 1 (Mst1), and induced apoptosis of K562 cells. The caspase-3 inhibitor Z-VAD (40 $\mu \mathrm{mol} / \mathrm{L})$ reduced imatinibinduced H2AX phosphorylation at Ser139 and Tyr142 and blocked imatinib-induced apoptosis of K562 cells. Imatinib (4 $\mu \mathrm{mol} / \mathrm{L})$ induced expression of Williams-Beuren syndrome transcription factor (WSTF), but not wild-type p53-induced phosphatase 1 (Wip1) in K562 cells.

Conclusion: The caspase-3/Mst1 pathway is required for H2AX C-terminal phosphorylation at Ser139 and Tyr142 and subsequent apoptosis in Bcr-Abl-positive K562 cells induced by imatinib.

Keywords: chronic myelogenous leukemia; imatinib; H2AX; phosphorylation; acetylation; apoptosis; caspase-3; mammalian STE20-like kinase 1 (Mst1); Z-VAD; Williams-Beuren syndrome transcription factor

Acta Pharmacologica Sinica (2012) 33: 551-557; doi: 10.1038/aps.2012.9; published online 5 Mar 2012

\section{Introduction}

Chronic myelogenous leukemia (CML) is a myeloproliferative disorder of the hematopoietic stem cells, caused by a $\mathrm{t}(9 ; 22)$ (q34; q11) translocation that generates a BCR-ABL fusion gene encoding a BCR-ABL fusion oncoprotein ${ }^{[1-3]}$. $\mathrm{BCR}-\mathrm{ABL}$ fusion protein possesses constitutively active tyrosine kinase activity, which plays an essential role in the initiation of CML. Multiple signaling pathways including Ras, Stat 5, Erk/MAPK, Jak2, PI3K, and others are activated by BCR-ABL, leading to excessive and uncontrolled proliferation of differentiated myeloid cells ${ }^{[4]}$. Clinically, CML has

\footnotetext{
* To whom correspondence should be addressed.

E-mail luchengrong@263.net (Cheng-rong LU); duanlianning@hotmail.com (Lian-ning DUAN) Received 2011-11-29 Accepted 2012-01-12
}

been successfully treated with imatinib mesylate, a selective small-molecule protein kinase inhibitor that specifically targets the oncogenic BCR-ABL fusion protein kinase, although the regulation mechanism involved in imatinib-induced CML cell apoptosis is only partially understood ${ }^{[5,6]}$. However, new difficulties have challenged clinicians. It has also been found that many CML patients have resistance to imatinib treatment because of a high frequency of BCR-ABL fusion gene mutations ${ }^{[5-7]}$. Thus, identifying the signaling pathways or biochemical mediators of imatinib-induced CML cell death may help to develop innovative strategies to overcome imatinib resistance.

Histone variant $\mathrm{H} 2 \mathrm{AX}$ is a major regulator of the cellular response to DNA damage and is associated with cell death ${ }^{[8,9]}$. Increasing evidence has demonstrated that the function of $\mathrm{H} 2 \mathrm{AX}$ is mainly regulated by its C-terminal phosphorylation 
at Ser139 ${ }^{[10,11]}$. The phosphorylated form of H2AX at Ser139 has also been referred to as $\gamma \mathrm{H} 2 \mathrm{AX}^{[12]}$. We have previously reported that $\gamma \mathrm{H} 2 \mathrm{AX}$ is required for DNA degradation mediated by caspase-activated DNase (CAD) in apoptotic cells ${ }^{[9]}$. Recently C-terminal Tyr142 of H2AX has been found to be a phosphorylation site ${ }^{[13,14]}$. Tyr142 phosphorylation is not a prerequisite for Ser139 phosphorylation but cooperates to regulate DNA damage repair or apoptosis. In vitro and in vivo experiments have both already provided compelling evidence that H2AX phosphorylation is related to tumor development ${ }^{[15-17]}$. It has been reported that deletion of H2AX attenuates the apoptotic response of gastrointestinal stromal tumors (GISTs) to imatinib and induces blast crisis of CML in a transgenic mouse model ${ }^{[16,18]}$. Taken together, these published data indicate that H2AX plays an important role in regulation of tumor cell apoptosis and acts as a novel human tumor suppressor protein. However, it is still unknown how H2AX phosphorylation at Ser139 and Tyr142 is regulated in CML cells. Therefore, identification of the signaling pathways involved in imatinib-induced $\mathrm{H} 2 \mathrm{AX}$ phosphorylation and apoptosis may provide new molecular targets for imatinibresistant CML or other cancer therapy.

In this study, we used imatinib to induce apoptosis of BCR-ABL-positive K562 cells and investigated the signaling pathways involved in regulation of H2AX phosphorylation (Ser139/Tyr142). We demonstrated that imatinib induced strong phosphorylation of H2AX (Ser139/Tyr142) in a timeand dose-dependent manner. However, H2AX acetylation (Lys 5) was not induced by imatinib. Caspase-3 and its downstream Mst1 could be activated by imatinib during apoptosis, coinciding with H2AX phosphorylation at Ser139 and Tyr142. Inhibition of the caspase-3/Mst1 pathway with caspase-3 inhibitor Z-VAD reduced H2AX phosphorylation at Ser139 and Tyr142 and blocked K562 cell apoptosis. In addition, we found that expression of WSTF, which is reported to regulate $\mathrm{H} 2 \mathrm{AX}$ phosphorylation at Tyr142, was increased by imatinib. However, expression of Wip1, which has been shown to dephosphorylate H2AX at Ser139, was not affected by imatinib. Overall, these data show that the caspase-3/Mst1 pathway is required for imatinib-induced H2AX C-terminal phosphorylation (Ser139 and Tyr142) and subsequent apoptosis of Bcr-Abl-positive K562 cells.

\section{Materials and methods Reagents}

Imatinib mesylate and caspase-3 inhibitor Z-VAD were purchased from Axon Medchem (Groningen, Netherlands) and Calbiochem (La Jolla, CA, USA), respectively. The antibodies against $\gamma \mathrm{H} 2 \mathrm{AX}$, phosphorylated H2AX (Y142), acetylated H2AX (Lys 5) (aH2AX), H2AX, Mst1, active Mst1, and WSTF were obtained from Millipore (Billerica, MA, USA). The antibody to detect $\beta$-actin was from Sigma-Aldrich (St Louis, MO, USA). The antibodies against caspase-3, activated caspase-3 (cleaved caspase-3 fragment) and Wip1 were obtained from
Santa Cruz Biotechnology (Santa Cruz, CA, USA).

\section{Cell culture and chemical drug treatments}

K562 cells were cultured in RPMI-1640 medium supplemented with $10 \%$ fetal bovine serum (FBS) in a $37^{\circ} \mathrm{C} 5 \% \mathrm{CO}_{2}$ incubator. Before each experiment, K562 cells $\left(5 \times 10^{6}\right)$ were seeded in $10-\mathrm{cm}$ dishes and treated for various times with culture medium supplemented with imatinib mesylate or with Z-VAD $1 \mathrm{~h}$ before treatment with imatinib mesylate.

\section{Observation of apoptotic cell morphology}

K562 cells after treatment with imatinib mesylate at different concentrations for various times were observed by inverted microscopy (Olympus, Tokyo, Japan). To detect chromatin condensation, K562 cells after imatinib mesylate treatment were stained with DAPI (Cell Apoptosis DAPI Detection Kit; KeyGen Biotech, Nanjing, China). All samples were viewed with a fluorescence microscope (Olympus).

\section{Total cellular protein or histone extraction and Western blot analysis}

Cellular proteins were extracted after drug treatments by disrupting the cells in lysis buffer $(50 \mathrm{mmol} / \mathrm{L}$ Tris- $\mathrm{HCl}$, $\mathrm{pH} 7.4,1 \% \mathrm{NP}-40,0.25 \%$ sodium deoxycholate, $150 \mathrm{mmol} / \mathrm{L}$ $\mathrm{NaCl}, 1 \mathrm{mmol} / \mathrm{L}$ EGTA, $1 \mathrm{mmol} / \mathrm{L} \mathrm{Na}_{3} \mathrm{VO}_{4}, 1 \mathrm{mmol} / \mathrm{L} \mathrm{NaF}$, $1 \mathrm{mg} / \mathrm{mL}$ aprotinin, $1 \mathrm{mg} / \mathrm{mL}$ leupeptin, $1 \mathrm{mg} / \mathrm{mL}$ pepstatin, and $1 \mathrm{mmol} / \mathrm{L} \mathrm{PMSF}$ ). To extract the histones after drug treatment, cells were lysed in NETN buffer $(150 \mathrm{mmol} / \mathrm{L} \mathrm{NaCl}$, $1 \mathrm{mmol} / \mathrm{L}$ EDTA, $20 \mathrm{mmol} / \mathrm{L}$ Tris, $\mathrm{pH} 8$, and 0.5\% NP-40) and centrifuged for $5 \mathrm{~min}$. The histones were extracted from the pellets with $0.1 \mathrm{~mol} / \mathrm{L} \mathrm{HCl}^{[10]}$. The protein samples were resolved by SDS-PAGE and transferred to polyvinylidene difluoride membranes. The membranes were blocked at room temperature for $1 \mathrm{~h}$ with $5 \%$ nonfat milk in Tris-buffered saline containing Tween 20 (TBST). Primary antibodies were incubated with membranes at $4{ }^{\circ} \mathrm{C}$ overnight. Membranes were incubated with the appropriate secondary antibody in TBST for $1 \mathrm{~h}$ at room temperature. Proteins were detected by enhanced chemiluminescence (Amersham Biosciences, Bucks, UK).

\section{Flow cytometry}

Imatinib-induced apoptosis of K562 cells was examined using a PE Annexin V Apoptosis Detection Kit (BD Biosciences, San Jose, CA, USA) according to the protocol provided. Cells were washed once with RPMI-1640 medium containing serum, and incubated with Annexin V-conjugated PE. Apoptosis was analyzed by flow cytometer (FACS-Calibur; Becton Dickinson, San Jose, CA, USA).

\section{Statistical analysis}

Data are expressed as the mean \pm SD from three independent determinations, and significance was assessed using a $t$ test and one-way ANOVA. Differences were considered significant at $P<0.01$. 


\section{Results}

Imatinib induces H2AX C-terminal phosphorylation and apoptosis Human K562 cells are BCR-ABL-positive CML cells ${ }^{[19]}$. Imatinib inhibits the kinase activity of BCR-ABL, leading to inhibition of K562 cell growth and apoptosis. Previous data have shown that H2AX C-terminal phosphorylation is required for tumor cell apoptosis ${ }^{[17,20,21]}$. Hence, we investigated whether imatinib could induce H2AX C-terminal phosphorylation in CML cells. Histones were extracted from K562 cells after imatinib treatment at the indicated time and dose and Western blot analysis was conducted. The data demonstrated that treatment with imatinib for 24 or $48 \mathrm{~h}$ induced H2AX C-terminal phosphorylation at Ser139 and Tyr142 in a dose-dependent manner (Figure 1A). At the same time, we also detected H2AX acetylation and found that imatinib did not induce $\mathrm{H} 2 \mathrm{AX}$ acetylation (Lys 5) in K562 cells (Figure 1A). Our data showed that H2AX acetylation appeared not to affect H2AX phospho- rylation (Ser139/Tyr142). However, the reason why K562 cells maintained such a high endogenous level of acetylated H2AX (Lys 5) and how acetylated H2AX (Lys 5) functioned is an interesting issue that requires further investigation (Figure 1A).

We also observed that K562 cell apoptosis (cell shrinking) was triggered by imatinib in a dose-dependent manner (Figure 1B), following a parallel response with $\mathrm{H} 2 \mathrm{AX}$ phosphorylation (Ser139/Tyr142) (Figure 1A). These data suggested that H2AX C-terminal phosphorylation was related to apoptosis of CML cells, and supported the previous suggestion that H2AX C-terminal phosphorylation is involved in tumor cell apoptosis.

\section{Imatinib triggers activation of caspase-3 and its downstream} Mst1

Caspase-3 has been identified as a key mediator of apoptosis of mammalian cells, and its activation is believed to be a hall-
A

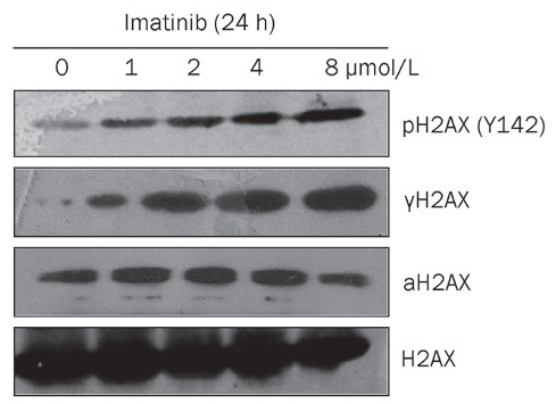

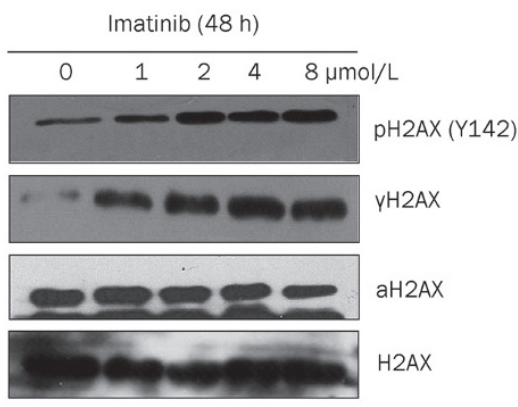

B

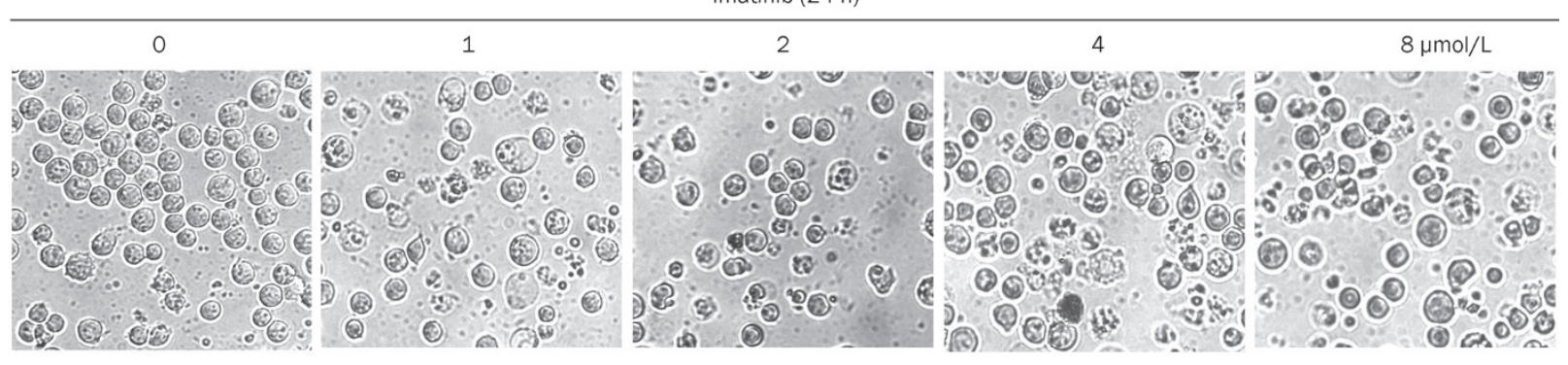

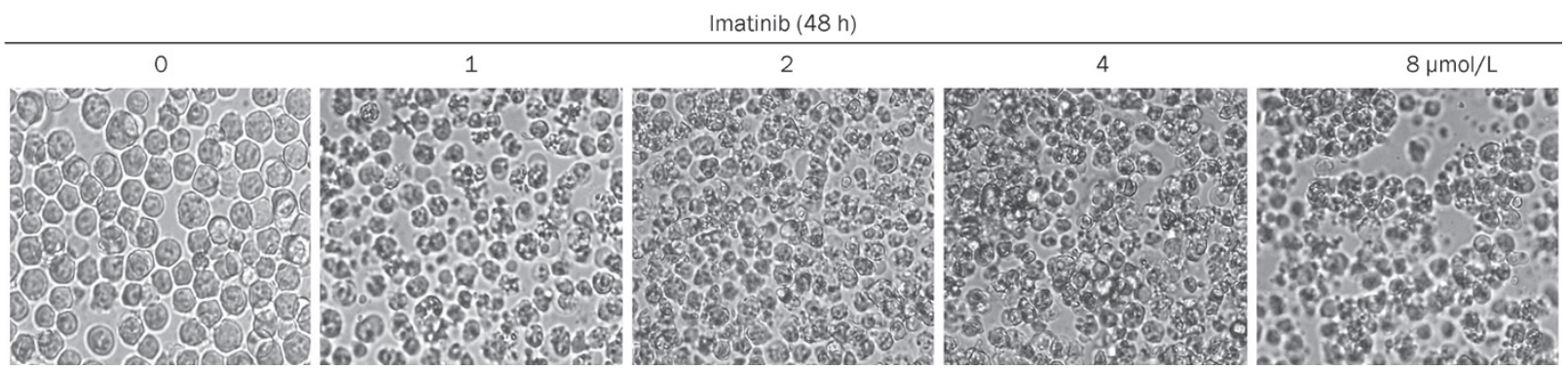

Figure 1. Imatinib induces $\mathrm{H} 2 \mathrm{AX}$ phosphorylation at Ser139 and Tyr142 and apoptosis. (A) K562 cells were treated with different concentrations of imatinib as indicated for $24 \mathrm{~h}$ (left panel) and $48 \mathrm{~h}$ (right panel). Cells not treated with imatinib served as negative controls. The histones were extracted and resolved by 15\% SDS-PAGE followed by Western blot analysis with antibodies against yH2AX (phosphorylated H2AX at Ser139), pH2AX (Y142) (phosphorylated H2AX at Tyr142), aH2AX (acetylated H2AX at Lys 5) and total H2AX. (B) As for (A), K562 cells after treatment with imatinib at the indicated time and dose were observed by microscopy (original magnification: $\times 400$ ). 
mark of apoptosis, and to regulate chromatin condensation by its downstream $\mathrm{CAD}^{[22]}$. Mst1, another caspase-3 downstream protein, has recently been reported to phosphorylate H2AX at Ser139 directly in HEK 293 cells, resulting in formation of $\gamma \mathrm{H} 2 \mathrm{AX}$, which regulates apoptosis ${ }^{[23]}$. To investigate whether the caspase-3/Mst1 signaling pathway is involved in regulation of C-terminal phosphorylation of H2AX in CML cells, we studied the effect of imatinib on activation of the caspase-3/ Mst1 pathway, using Western blot analysis. We indicated that caspase-3 and Mst1 were dramatically activated after 24 or $48 \mathrm{~h}$ imatinib treatment in a dose-dependent manner (Figure 2A). Caspase-3 (34 kDa) was cleaved to produce the active fragment $(17 \mathrm{kDa})$. Simultaneously, Mst1 $(54 \mathrm{kDa})$ was cleaved by activated caspase- 3 to form the active Mst1 fragment $(34 \mathrm{kDa})$. Together with these data, we demonstrated that caspase- 3 and Mst1 activation were triggered by imatinib (Figure 2A), following a similar response as that for H2AX C-terminal phosphorylation induced by imatinib (Figure 1A). These results suggest that the caspase-3/Mst1 pathway contributes to C-terminal phosphorylation of H2AX. To confirm that imatinib induces apoptosis, we stained K562 cells with DAPI to observe chromatin condensation. The data clearly showed that imatinib induced strong apoptosis of K562 cells in a time-dependent manner (Figure 2B).
Caspase-3/Mst1 pathway is required for imatinib-induced H2AX phosphorylation and apoptosis

The data presented in Figures 1 and 2 indicated that imatinib might induce H2AX C-terminal phosphorylation (Ser139 and Tyr142) via the caspase-3/Mst1 pathway during K562 cell apoptosis. To confirm this, we used caspase-3 inhibitor Z-VAD to block caspase-3/Mst1 signaling in K562 cells and then detected H2AX C-terminal phosphorylation and apoptosis induced by imatinib. K562 cells treated with imatinib alone or together with Z-VAD were harvested for Western blotting and flow cytometry. Imatinib triggered caspase- 3 and Mst1 activation in a time-dependent manner (Figure 3, bottom left). At the same time, phosphorylation of H2AX at Ser139/ Tyr142 was induced (Figure 3, top left). To investigate the action of the caspase-3/Mst1 pathway in regulation of H2AX phosphorylation at Ser139/Tyr142 and apoptosis of K562 cells, Z-VAD was used to treat K562 cells with imatinib. We found that Z-VAD inhibited the cleavage of caspase- 3 and Mst1 to form their active fragments. That is to say, Z-VAD inhibited imatinib-induced activity of caspase-3, leading to inhibition of Mst1 activity (Figure 3, bottom right). Simultaneously, imatinib-induced phosphorylation of Ser139 and Tyr142 in H2AX was inhibited by Z-VAD (Figure 3, top right).

Furthermore, we also detected the function of the caspase-3/
A

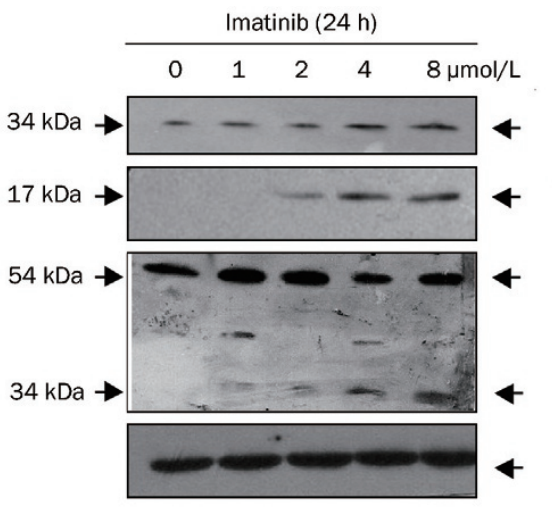

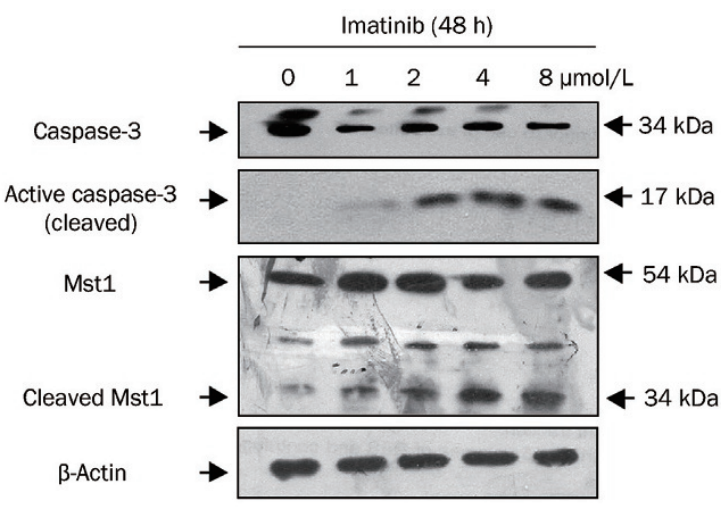

Imatinib $(4 \mu \mathrm{mol} / \mathrm{L})$

B

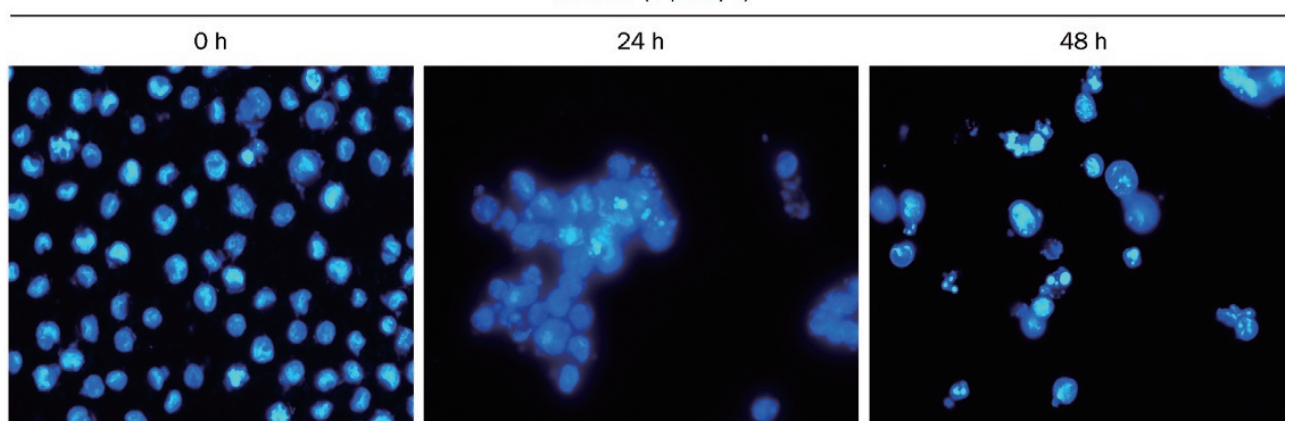

Figure 2. Imatinib induces caspase-3 and Mst1 activation and apoptosis. (A) K562 cells were treated with imatinib at the indicated concentrations for $24 \mathrm{~h}$ (left panel) or $48 \mathrm{~h}$ (right panel) and harvested for Western blot analysis to detect caspase-3 (full length, $34 \mathrm{kDa}$ ), cleavage of caspase-3 (17 kDa fragment), Mst1 (full length, $54 \mathrm{kDa}$ ), and cleaved Mst1 (34 kDa). $\beta$-Actin was also detected as a loading control. (B) K562 cells were treated with imatinib $(4 \mu \mathrm{mol} / \mathrm{L})$ for 0,24 , or $48 \mathrm{~h}$ and stained with DAPI for observation by immunofluorescence microscopy (original magnification: $\times 400$ ). 

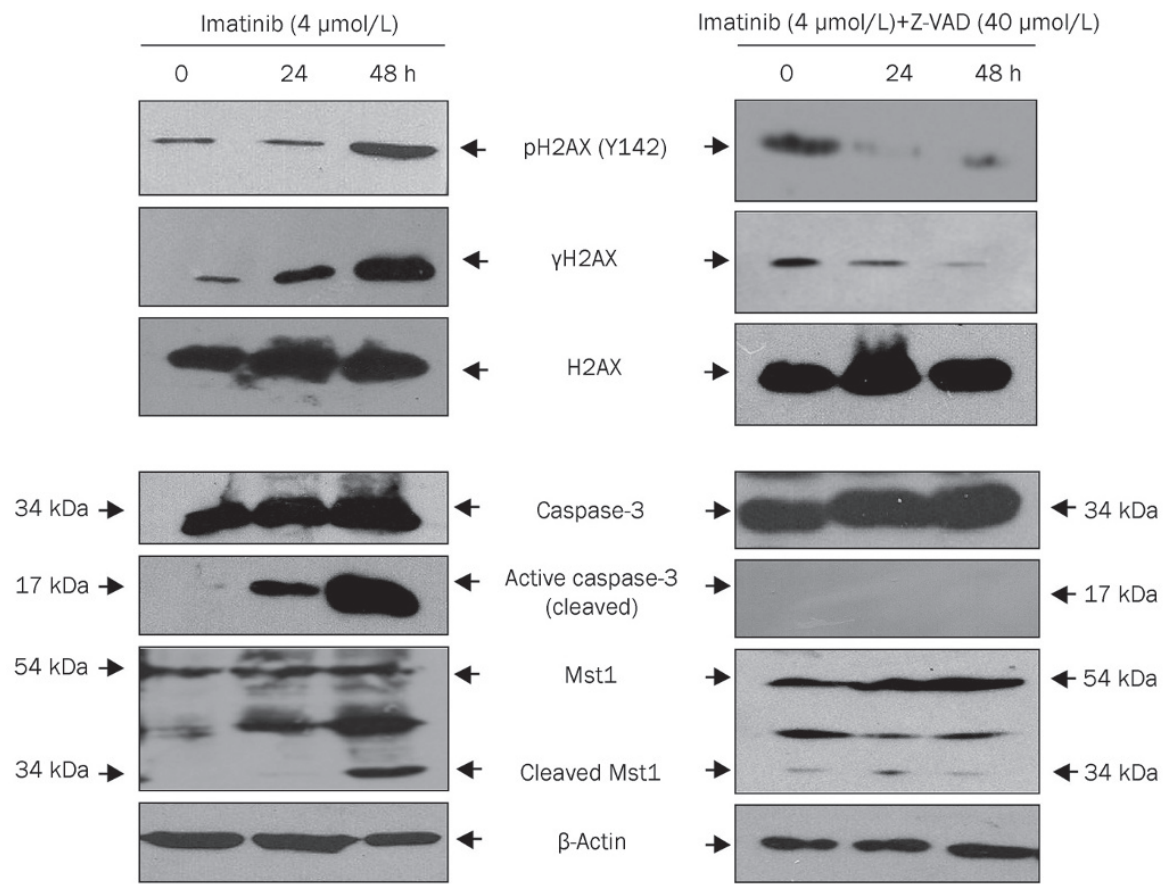

Figure 3. Caspase-3/Mst1 pathway is involved in imatinib-induced $\mathrm{H} 2 \mathrm{AX}$ phosphorylation at Ser139 and Tyr142. K562 cells were treated with imatinib (left panels) and imatinib with Z-VAD (right panel) for the indicated time. The extracted whole proteins were resolved by $10 \%$ SDS-PAGE followed by Western blot analysis with antibodies against caspase-3 (full length, $34 \mathrm{kDa}$ ), cleaved caspase-3 (17 kDa fragment), Mst1 (full length, $54 \mathrm{kDa}$ ), and cleaved Mst1 (34 $\mathrm{kDa}$ ) (bottom, left panel and right panel). The extracted histones were analyzed by Western blotting to detect phosphorylation of $\mathrm{H} 2 \mathrm{AX}$ [pH2AX (Y142), $\mathrm{yH} 2 \mathrm{AX}$, and total H2AX] (top left and right panels).

Mst1 pathway in regulation of K562 cell apoptosis induced by imatinib. Flow cytometry showed that imatinib effectively induced apoptosis of $\mathrm{K} 562$ cells, characterized by the percentage of early apoptotic cells, in a time-dependent manner (Figure 4). Z-VAD strongly reduced imatinib-induced apoptosis of K562 cells (Figure 4).

Overall, these data provided strong evidence that H2AX phosphorylation (Ser139/Tyr142) and apoptosis of K562 cells induced by imatinib is regulated by the caspase-3/Mst1 signaling pathway.
Imatinib induces Tyr142 phosphorylation of H2AX by increasing WSTF but not Wip1 expression

Wip1 is a nuclear oncogenic type $2 \mathrm{C}$ protein phosphatase that is amplified in many human tumors, such as breast cancer, ovarian clear cell carcinoma, and medulloblastoma ${ }^{[24,25]}$. Previous data have indicated that Wip1 directly dephosphorylates $\gamma \mathrm{H} 2 \mathrm{AX}$ (Ser139) and regulates DNA damage response. Hence, we investigated whether imatinib induced H2AX phosphorylation (Ser139) by inhibiting expression of Wip1. We showed that imatinib did not increase Wip1 expression in K562 cells (Figure 5A).

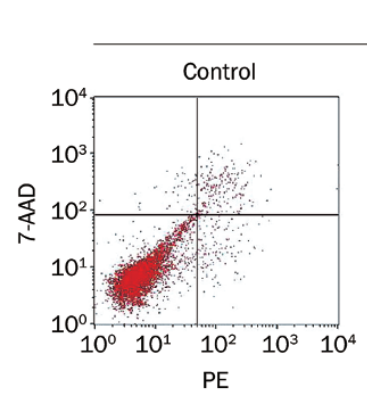

Imatinib (4 $\mu \mathrm{mol} / \mathrm{L})$
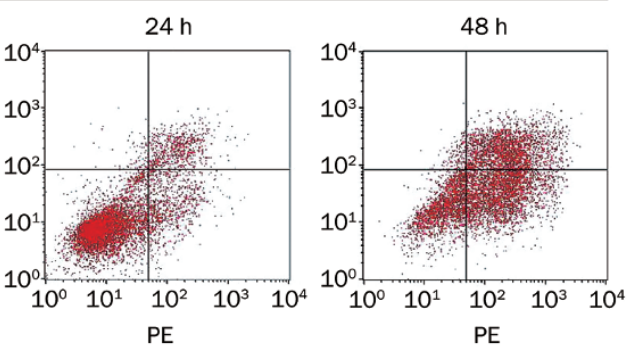

Imatinib $(4 \mu \mathrm{mol} / \mathrm{L})+Z-V A D(40 \mu \mathrm{mol} / \mathrm{L})$
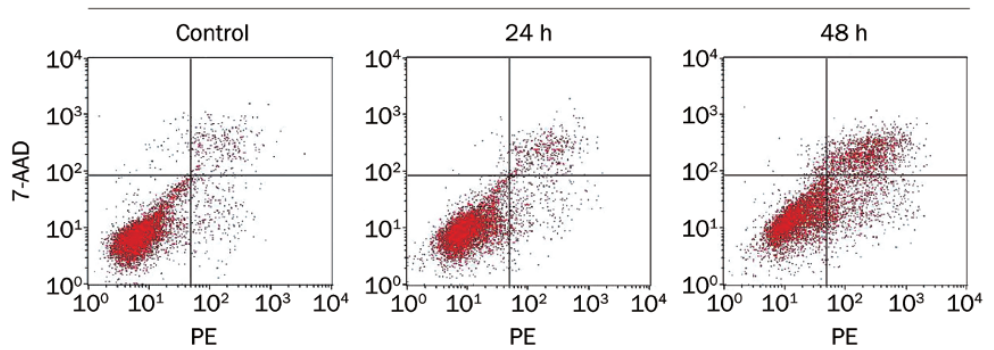

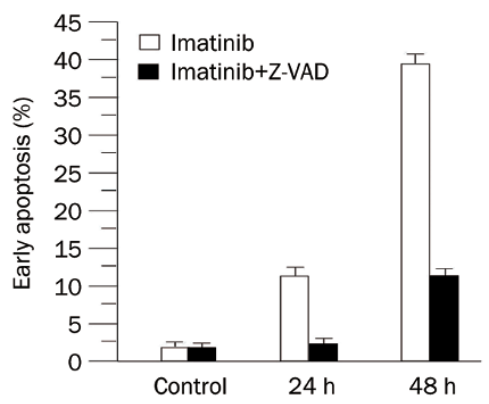

Figure 4. Caspase- 3 is required for apoptosis of $\mathrm{K} 562$ cells. K562 cells were treated with $4 \mu \mathrm{mol} / \mathrm{L}$ imatinib (top left panels) and $4 \mu \mathrm{mol} / \mathrm{L}$ imatinib with $40 \mu \mathrm{mol} / \mathrm{L} \mathrm{Z-VAD} \mathrm{(bottom}$ left panels). Cells were harvested at the indicated time and subjected to flow cytometry after Annexin V-conjugated PE staining to assess early apoptosis (PE Annexin $\mathrm{V}$ positive and 7-AAD negative) (right bottom area). The mean \pm SD of three independent determinations are shown (right panel) $(P<0.01)$. 
A

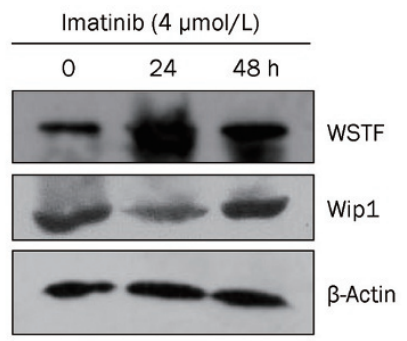

B

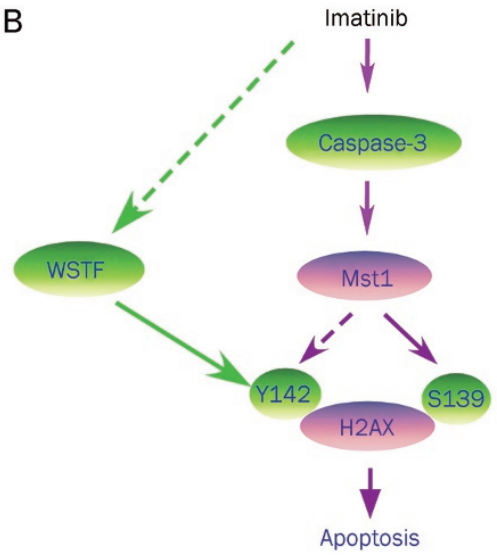

Figure 5. (A) WSTF regulates imatinib-induced Tyr142 phosphorylation of $\mathrm{H} 2 \mathrm{AX}$. K562 cells were treated with $4 \mu \mathrm{mol} / \mathrm{L}$ imatinib at the indicated time. The extracted whole proteins were subjected to Western blot analysis with antibodies against WSTF, Wip1, and $\beta$-actin. (B) A regulation model of H2AX phosphorylation (C-terminal) during imatinib-induced apoptosis. Once caspase- 3 activation is triggered by imatinib and its down stream Mst1 will be cleaved and activated. Active Mst1 can regulate H2AX phosphorylation at Ser139 (S139) and Tyr142 (Y142), which is involved in apoptosis. Simultaneously, imatinib can also increases Tyr142 phosphorylation of $\mathrm{H} 2 \mathrm{AX}$ by increasing WSTF protein level to regulate apoptosis. The dotted arrows indicate the regulation mechanisms that remain to be characterized further.

Another protein, WSTF, a component of the WICH complex (WSTF-ISWI ATP-dependent chromatin-remodeling complex) was found to regulate Tyr142 phosphorylation of H2AX ${ }^{[14]}$. We indicated that imatinib actually increased WSTF level in K562 cells (Figure 5A). This is a novel finding, suggesting that WSTF protein at least partially contributed to imatinibinduced H2AX phosphorylation (Tyr142) to regulate apoptosis. How imatinib regulates expression of WSTF or whether the caspase-3/Mst1 pathway is also involved in regulation of WSTF protein level requires further investigation.

\section{Discussion}

Histone $\mathrm{H} 2 \mathrm{AX}$ is a variant of histone $\mathrm{H} 2 \mathrm{~A}$ that is ubiquitously expressed throughout the genome, and has been designated as the histone guardian of the genome ${ }^{[26]}$. Deficiency of histone $\mathrm{H} 2 \mathrm{AX}$ decreases genomic stability and increases tumor susceptibility of normal cells and tissues, suggesting that it might act as a tumor suppressor ${ }^{[27,28]}$. Increasing evidence, along with our present results show that the function of
$\mathrm{H} 2 \mathrm{AX}$ involved in regulation of apoptosis is mediated by its C-terminal phosphorylation. We previously have demonstrated that H2AX phosphorylation at Ser139 plays a key role in regulation of apoptosis ${ }^{[9,10]}$. Mutation of Ser139 in H2AX to block Ser139 phosphorylation greatly inhibited apoptosis. Recently, Tyr142 in the C terminus of H2AX was found to be regulated by WSTF during apoptosis. Tyr142 phosphorylation has been confirmed as not essential for Ser139 phosphorylation, but it might cooperate to regulate DNA damage repair or apoptosis ${ }^{[14]}$. We found that WSTF protein level in K562 cells was increased by imatinib treatment, indicating that imatinib induced Tyr142 phosphorylation, partially dependent on WSTF. However, we excluded the possibility of Wip1 involved in imatinib-induced phosphorylation of H2AX at Ser139. To summarize our present results, we suggest a regulation model of H2AX C-terminal phosphorylation during imatinib-induced apoptosis. The model demonstrates that imatinib can induce both Ser139 and Tyr142 phosphorylation, and apoptosis in K562 cells through the caspase-3/Mst1 pathway (Figure 5B). Furthermore, the imatinib-induced increase in WSTF level was at least partially involved in phosphorylation of H2AX at Tyr142 (Figure 5B). Our previous work has shown that H2AX phosphorylated at Ser139 ( $\gamma \mathrm{H} 2 \mathrm{AX}$ ) regulates DNA fragmentation triggered by CAD in apoptotic cells ${ }^{[9]}$. However, how the Tyr142 phosphorylation of H2AX cooperates with Ser139 phosphorylation of H2AX to regulate apoptosis is currently unknown in detail.

Our data confirmed the importance of H2AX in apoptosis of CML cells. Interestingly, the H2AX gene maps to chromosome 11q23, a region which is frequently altered in mantle cell lymphoma and $\mathrm{CML}^{[2]}$. Furthermore, H2AX deficiency promotes B-cell tumorigenesis ${ }^{[2]}$. The data from the transgenic mouse model of CML further demonstrate that H2AX functions as a tumor suppressor, and low expression of H2AX promotes the blast crisis of $\mathrm{CML}^{[18]}$. H2AX dysfunction could make animals more sensitive to carcinogenic factors. Apparently, the function of H2AX as a tumor suppressor is related to its regulation of apoptosis. Carcinogenic factors usually induce DNA double-strand breaks (DSBs) in the genome, and cells themselves can trigger repair of these DSBs. Once DNA damage such as DSBs cannot be repaired, cells have to choose the apoptotic fate to protect the organism. If the apoptotic mechanism is prevented because of loss of H2AX function, the cells bearing an increasing number of DSBs possibly face a high risk of malignant mutations in the genome and therefore are easier to tumorigenesis.

CML is a clonal disease of hematopoietic stem cells that is characterized by BCR-ABL fusion protein, which has constitutive tyrosine kinase activity and is essential for the pathogenesis of the diseas ${ }^{[3]}$. Imatinib, an ATP-competitive selective inhibitor of BCR-ABL, has emerged as the lead compound for clinical development against CML because of its unprecedented efficacy ${ }^{[1]}$. However, the mechanism of apoptosis induced by imatinib is not known well, which prevents effective treatment of CML, especially in patients who develop imatinib resistance. H2AX has been found to be expressed at 
low levels in GISTs ${ }^{[16]}$. Low levels of $\gamma \mathrm{H} 2 \mathrm{AX}$ destroy the balance between growth and apoptosis of tumor cells, resulting in uncontrolled proliferation. Therefore, inhibition of H2AX phosphorylation is one possible reason why many patients with tumors develop resistance to radiotherapy and chemotherapy, including imatinib treatment. To the best of our knowledge, our study is the first to reveal the signal transduction pathway which regulates H2AX phosphorylation (Ser139/Tyr142), and to demonstrate the importance of H2AX phosphorylation in apoptosis of CML cells. Overall, these data provided mechanistic insights into imatinib-induced tumor cell apoptosis and suggested H2AX as a novel target in cancer therapy.

\section{Acknowledgements}

This work was supported by the National Natural Science Foundation of China (№ 30770430 and 81070424).

\section{Author contribution}

Yan-jun ZHANG performed most of experiments and analyzed the results. Cheng-rong LU, Yan-jun ZHAG, and Lianning DUAN designed the study. Cheng-rong LU wrote the manuscript, which was revised by Feng ZHU and Lian-ning DUAN. Feng ZHU and Mei XUE also provided support and general guidance for this work. Yan CAO and Shu YAN performed flow cytometry analysis for apoptosis. Yuan LUO, Rong-feng BAO, and Zhe WANG performed morphological observation of apoptotic cells.

\section{References}

1 Brauer KM, Werth D, von Schwarzenberg K, Bringmann A, Kanz L, Grunebach F, et al. BCR-ABL activity is critical for the immunogenicity of chronic myelogenous leukemia cells. Cancer Res 2007; 67: 5489-97.

2 Goldman JM, Melo JV. Chronic myeloid leukemia - advances in biology and new approaches to treatment. N Engl J Med 2003; 349: 1451-64.

3 Goldman JM, Melo JV. Targeting the BCR-ABL tyrosine kinase in chronic myeloid leukemia. N Engl J Med 2001; 344: 1084-6.

4 Yoshida C, Yoshida F, Sears DE, Hart SM, Ikebe D, Muto A, et al. Bcr$\mathrm{Abl}$ signaling through the $\mathrm{PI}-3 / \mathrm{S} 6$ kinase pathway inhibits nuclear translocation of the transcription factor Bach2, which represses the antiapoptotic factor heme oxygenase-1. Blood 2007; 109: 1211-9.

5 Strout MP, Schatz DG. Imatinib resistance and progression of CML to blast crisis: somatic hypermutation AIDing the way. Cancer Cell 2009; 16: $174-6$.

6 Lu Z, Jin Y, Chen C, Li J, Cao Q, Pan J. Pristimerin induces apoptosis in imatinib-resistant chronic myelogenous leukemia cells harboring T315I mutation by blocking NF-kappaB signaling and depleting BcrAbl. Mol Cancer 2010; 9: 112.

7 Deguchi Y, Kimura S, Ashihara E, Niwa T, Hodohara K, Fujiyama Y, et al. Comparison of imatinib, dasatinib, nilotinib and INNO-406 in imatinib-resistant cell lines. Leuk Res 2008; 32: 980-3.

8 Rogakou EP, Pilch DR, Orr AH, Ivanova VS, Bonner WM. DNA doublestranded breaks induce histone H2AX phosphorylation on serine 139. J Biol Chem 1998; 273: 5858-68.

9 Lu C, Zhu F, Cho YY, Tang F, Zykova T, Ma WY, et al. Cell apoptosis: requirement of $\mathrm{H} 2 \mathrm{AX}$ in DNA ladder formation, but not for the activation of caspase-3. Mol Cell 2006; 23: 121-32.

10 Lu C, Shi Y, Wang Z, Song Z, Zhu M, Cai Q, et al. Serum starvation induces $\mathrm{H} 2 \mathrm{AX}$ phosphorylation to regulate apoptosis via p38 MAPK pathway. FEBS Lett 2008; 582: 2703-8.

11 Cheung P, Allis CD, Sassone-Corsi P. Signaling to chromatin through histone modifications. Cell 2000; 103: 263-71.

12 Burma S, Chen BP, Murphy M, Kurimasa A, Chen DJ. ATM phosphorylates histone $\mathrm{H} 2 \mathrm{AX}$ in response to DNA double-strand breaks. J Biol Chem 2001; 276: 42462-7.

13 Cook PJ, Ju BG, Telese F, Wang X, Glass CK, Rosenfeld MG. Tyrosine dephosphorylation of $\mathrm{H} 2 \mathrm{AX}$ modulates apoptosis and survival decisions. Nature 2009; 458: 591-6.

14 Xiao A, Li H, Shechter D, Ahn SH, Fabrizio LA, Erdjument-Bromage H, et al. WSTF regulates the H2AX DNA damage response via a novel tyrosine kinase activity. Nature 2009; 457: 57-62.

15 Novik KL, Spinelli JJ, Macarthur AC, Shumansky K, Sipahimalani P, Leach S, et al. Genetic variation in H2AFX contributes to risk of nonHodgkin lymphoma. Cancer Epidemiol Biomarkers Prev 2007; 16: 1098-106.

16 Liu Y, Tseng M, Perdreau SA, Rossi F, Antonescu C, Besmer P, et al. Histone $\mathrm{H} 2 \mathrm{AX}$ is a mediator of gastrointestinal stromal tumor cell apoptosis following treatment with imatinib mesylate. Cancer Res 2007; 67: 2685-92.

17 Bonner WM, Redon CE, Dickey JS, Nakamura AJ, Sedelnikova OA, Solier S, et al. yH2AX and cancer. Nat Rev Cancer 2008; 8: 957-67.

18 Nagamachi A, Yamasaki N, Miyazaki K, Oda H, Miyazaki M, Honda Z. et al. Haploinsufficiency and acquired loss of Bcl11b and H2AX induces blast crisis of chronic myelogenous leukemia in a transgenic mouse model. Cancer Sci 2009; 100: 1219-26.

19 Jacquel A, Herrant M, Legros L, Belhacene N, Luciano F, Pages G, et al. Imatinib induces mitochondria-dependent apoptosis of the BcrAbl-positive $\mathrm{K} 562$ cell line and its differentiation toward the erythroid lineage. FASEB J 2003; 17: 2160-2.

20 Cha H, Lowe JM, Li H, Lee JS, Belova GI, Bulavin DV, et al. Wip1 directly dephosphorylates gamma-H2AX and attenuates the DNA damage response. Cancer Res 2010; 70: 4112-22.

21 Liu Y, Parry JA, Chin A, Duensing S, Duensing A. Soluble histone H2AX is induced by DNA replication stress and sensitizes cells to undergo apoptosis. Mol Cancer 2008; 7: 61.

22 Li P, Nijhawan D, Wang X. Mitochondrial activation of apoptosis. Cell 2004; 116: S57-9.

23 Wen W, Zhu F, Zhang J, Keum YS, Zykova T, Yao K, et al. MST1 promotes apoptosis through phosphorylation of histone H2AX. J Biol Chem 2010; 285: 39108-16.

24 Macurek L, Lindqvist A, Voets 0 , Kool J, Vos HR, Medema RH. Wip1 phosphatase is associated with chromatin and dephosphorylates gammaH2AX to promote checkpoint inhibition. Oncogene 2010; 29: 2281-91.

25 Moon SH, Lin L, Zhang X, Nguyen TA, Darlington Y, Waldman AS, et al. Wildtype p53-induced phosphatase 1 dephosphorylates histone variant $\mathrm{Y}-\mathrm{H} 2 \mathrm{AX}$ and suppresses DNA double strand break repair. J Biol Chem 2010; 285: 12935-47.

26 Kouzarides T. Chromatin modifications and their function. Cell 2007; 128: 693-705.

27 Zhu F, Zykova TA, Peng C, Zhang J, Cho YY, Zheng D, et al. Phosphorylation of H2AX at Ser139 and a new phosphorylation site Ser16 by RSK2 decreases H2AX ubiquitination and inhibits cell transformation. Cancer Res 2011; 71: 393-403.

28 Wu CY, Kang HY, Yang WL, Wu J, Jeong YS, Wang J, et al. Critical role of monoubiquitination of histone $\mathrm{H} 2 \mathrm{AX}$ protein in histone $\mathrm{H} 2 \mathrm{AX}$ phosphorylation and DNA damage response. J Biol Chem 2011; 286: 30806-15.

29 Klemm L, Duy C, lacobucci I, Kuchen S, von Levetzow G, Feldhahn N, et al. The B cell mutator AID promotes B lymphoid blast crisis and drug resistance in chronic myeloid leukemia. Cancer Cell 2009; 16 : 232-45. 